\title{
Potential role of iron in repair of inflammatory demyelinating lesions
}

\author{
Nathanael J. Lee, ${ }^{1,2}$ Seung-Kwon Ha, ${ }^{1}$ Pascal Sati, ${ }^{1}$ Martina Absinta, ${ }^{1}$ Govind Nair, ${ }^{1}$ Nicholas J. Luciano, ${ }^{1}$ Emily C. Leibovitch, ${ }^{3}$ \\ Cecil C. Yen, ${ }^{4}$ Tracey A. Rouault, ${ }^{5}$ Afonso C. Silva, ${ }^{4}$ Steven Jacobson, ${ }^{3}$ and Daniel S. Reich ${ }^{1}$
}

${ }^{1}$ Translational Neuroradiology Section, National Institute of Neurological Disorders and Stroke, National Institutes of Health, Bethesda, Maryland, USA. ²Department of Neuroscience, Georgetown University Medical Center, Georgetown University, Washington, District of Columbia, USA. ${ }^{3}$ Viral Immunology Section, National Institute of Neurological Disorders and Stroke, National Institutes of Health, Bethesda, Maryland, USA. ${ }^{4}$ Cerebral Microcirculation Section, National Institute of Neurological Disorders and Stroke, National Institutes of Health, Bethesda, Maryland, USA. ${ }^{5}$ Section on Human Iron Metabolism, National Institute of Child Health and Human Development, National Institutes of Health, Bethesda, Maryland, USA.

\begin{abstract}
Inflammatory destruction of iron-rich myelin is characteristic of multiple sclerosis (MS). Although iron is needed for oligodendrocytes to produce myelin during development, its deposition has also been linked to neurodegeneration and inflammation, including in MS. We report perivascular iron deposition in multiple sclerosis lesions that was mirrored in 72 lesions from 13 marmosets with experimental autoimmune encephalomyelitis. Iron accumulated mainly inside microglia/ macrophages from 6 weeks after demyelination. Consistently, expression of transferrin receptor, the brain's main iron-influx protein, increased as lesions aged. Iron was uncorrelated with inflammation and postdated initial demyelination, suggesting that iron is not directly pathogenic. Iron homeostasis was at least partially restored in remyelinated, but not persistently demyelinated, lesions. Taken together, our results suggest that iron accumulation in the weeks after inflammatory demyelination may contribute to lesion repair rather than inflammatory demyelination per se.
\end{abstract}

\section{Introduction}

Multiple sclerosis (MS) is an inflammatory demyelinating disease of the central nervous system (CNS) affecting millions of people worldwide (1). One of the hallmarks of MS is loss of myelin, which is abundant in iron, an essential element that is required for oligodendrocytes to synthesize myelin during development (2-4). However, iron deposition has also been linked to neurodegeneration and inflammation, including in MS, via oxidative injury (5-9). Iron can be found in some MS lesions and is detectable with both magnetic resonance imaging (MRI) and, when tissue is available, histopathology $(5,10-18)$. Despite these observations, the spatiotemporal dynamics of iron deposition and the cellular pathways involved in intralesional iron accumulation in MS have not been elucidated. Fundamentally limiting our understanding of the role of iron in MS is that most knowledge derives from histopathology-based studies of patients with long disease duration, potentially many years after iron first appears. Characterizing the spatiotemporal patterns of iron deposition using a relevant animal model may help identify the timing, origin, and pathophysiological significance of intralesional iron deposition.

Experimental autoimmune encephalomyelitis (EAE) in the common marmoset (Callithrix jacchus) successfully recapitulates many pathological and radiological features of MS lesions, providing an opportunity to investigate how inflammatory demyelinating lesions develop, as well as the spatiotemporal dynamics of, and the

Conflict of interest: The authors have declared that no conflict of interest exists. Copyright: @ 2019, American Society for Clinical Investigation.

Submitted: December 14, 2018; Accepted: July 16, 2019; Published: September 9, 2019. Reference information: / Clin Invest. 2019;129(10):4365-4376.

https://doi.org/10.1172/JCI126809. involvement of iron in, lesion pathogenesis and evolution (19-22). Interestingly, a recent study demonstrated that iron deposition is one of the pathological hallmarks of marmoset EAE lesions (23), which highlights the relevance of marmoset EAE to investigate MS-like pathobiology involving iron.

Prior work suggests that dysregulated iron accumulation can be detrimental to the CNS in various ways. For example, iron-laden microglia tend to stay in a proinflammatory state $(24,25)$ and lose the ability to clear myelin debris in lesions, preventing oligodendrocytes or their precursors from entering the injured sites for remyelination and repair $(6,26)$. Furthermore, iron can cause oxidative stress, free radical toxicity, and eventually cell death via various cellular pathways $(8,9,23,27-31)$. These observations prompted iron chelation treatment trials in both MS (32-34) and EAE (35-38). However, most studies have yielded unpredictable and mixed results, potentially due to the imperfect characterization of the role and timing of iron accumulation in MS lesions and the mechanisms of iron regulation during the process of repair and remyelination in the CNS.

To address the role of iron in acute inflammatory demyelination, we studied white matter lesion formation in marmoset EAE. We used serial in vivo MRI, specifically proton-density weighted (PDw) MRI - which can detect demyelination - to characterize and date the formation of focal lesions in the white matter, and categorized the lesions based on their age $(21,39)$. We also looked for similar iron deposition patterns in human MS lesions. To determine the spatiotemporal deposition of intralesional iron in vivo, we used $\mathrm{T} 2^{*}$-weighted $\left(\mathrm{T} 2^{*} \mathrm{w}\right)$ MRI, which is sensitive to paramagnetic substances. We further characterized the lesions using histopathology, focusing on iron and its associated protein regulators, to identify differential expression throughout various stages of lesion development. 


\section{Results}

Iron is found in older EAE lesions inside perivascular microglia/macrophages, and in a similar pattern in MS lesions. In 13 marmosets, we analyzed 10 healthy white matter areas. We also analyzed 5 nondemyelinated inflammatory nodules (NDINs), which represent the earliest stage of lesion evolution, are identifiable only histopathologically, and feature vascular permeability and minimal perivascular inflammation but no visible change on in vivo structural MRI $(21,39)$. In addition, we analyzed 18 lesions that were 0-2 weeks old, 8 lesions that were 2-6 weeks old, 25 demyelinated lesions older than 6 weeks, and 14 remyelinated lesions older than 6 weeks. At least 3 different marmosets contributed data to each group. To investigate the spatiotemporal dynamics of iron deposition, we tracked lesion age using serial in vivo PDw MRI. With serial in vivo $\mathrm{T} 2{ }^{*} \mathrm{~W}$ MRI, we identified intralesional hypointense puncta (Figure 1A) and later studied their colocalization with iron deposition on histopathology, confirming that this pattern of signal loss is due to the paramagnetic properties of iron (Figure 1B).

Our data from 72 lesions show that iron is mainly found in lesions older than 6 weeks (Figure 2A, C-F): 33 of 39 lesions older than 6 weeks harbored iron, compared with only 2 of 33 younger lesions. The average age of iron ${ }^{+}$lesions was 19.5 weeks, whereas that of iron- lesions was 1.5 weeks (Figure 2C). Double-staining showed that iron is harbored inside $\mathrm{Iba}^{+}$microglia/macrophages (where it is closely associated with ferritin; Figure 2B), but not in $\mathrm{GFAP}^{+}$astrocytes, and it is mainly located around blood vessels.

We also evaluated in vivo brain scans of $39 \mathrm{MS}$ cases, observing, in $56 \%$, at least one lesion with strong intralesional focal hypointensity on $\mathrm{T} 2{ }^{*} \mathrm{~W}$ MRI, similar to the pattern observed in marmosets (Figure 1C). In cases with fewer than 20 focal white matter lesions at the time of the scan $(n=14)$, lesion counting showed that $6 \%$ of all focal white matter lesions (9 of 133) contained foci suggestive of iron deposition. Of 15 lesions analyzed from 4 MS brains donated to research, we found a similar pattern on ex vivo T2* ${ }^{*} \mathrm{MRI}$ in one lesion, where the focal hypointensity on MRI corresponded to iron deposition on DAB-Turnbull staining (Figure 1D).

Iron-regulating protein levels change throughout lesion progression. To investigate the pathophysiology of intralesional, intracellular iron accumulation, we measured the levels of key iron-regulating proteins, including transferrin receptor (TfR, a major iron influx protein), ferroportin ( $\mathrm{FpN}$, the main known cellular iron efflux channel), and hepcidin ( $\mathrm{HpC}$, a protein that binds and degrades FpN), using immunohistochemistry. TfR and $\mathrm{HpC}$, but not FpN, increased with lesion age and peaked when lesions were 0-2 weeks old (Figure 3, A-D), shortly before iron accumulation. Divalent metal transporter 1 (DMT-1), another iron influx protein, had a similar pattern (Figure 3A). To further elucidate the relationship between iron accumulation and increased levels of TfR, we investigated its specific localization by immunohistochemistry and in situ hybridization. We found that TfR levels increased in the endothelium of blood vessels within lesions (Supplemental Figure 1, A and B; supplemental material available online with this article; https://doi. org/10.1172/JCI126809DS1), and that TfR mRNA was expressed within cells in lesions, suggesting that it is synthesized de novo.

Iron is not associated with early, active inflammation. Previous studies have suggested that iron may contribute to inflammation and oxidative stress in the CNS $(5,6,23,27,29)$. Therefore, we tested the spatiotemporal correlation between iron deposition and known markers of those processes. We found that inflammatory cells, including Iba $1^{+}$microglia/macrophages, $\mathrm{MRP}^{+} 4^{+}$earlyactivated peripheral macrophages, and $\mathrm{CD}^{+} \mathrm{T}$ cells, as well as the oxidative stress marker superoxide dismutase (SOD), appeared at the very earliest stages of lesions (NDIN) and persisted throughout lesion evolution (Figure 4), whereas iron was mainly found in later stages (Figure 1A, Figure 2, A and C-F). This suggests that iron does not play an important role in early, active inflammatory processes related to demyelination, which occurs during the first 6 weeks $(21,39)$. Indeed, the density of inflammatory cells decreased once lesions were more than 6 weeks old.

Iron-regulating protein levels are different in demyelinated versus remyelinated lesions. Although numerous studies have focused on the proinflammatory role of iron in the CNS, it is also known that iron is essential for both developmental myelinogenesis and tissue repair $(3,4,9,40-43)$. Since we observed that iron is not associated with early inflammatory demyelination, we investigated whether iron-regulating protein levels are different in iron ${ }^{+}$lesions that are demyelinated compared with iron ${ }^{+}$lesions that show signs of repair and remyelination. We first identified repairing lesions using serial in vivo PDw MRI, marking lesions in which hyperintense signal partially or completely resolved over time (Supplemental Figure 2A). We then confirmed that this change in MR signal corresponds with myelin repair and repopulation of oligodendrocyte-lineage cells (Supplemental Figure 2, B-D). In such lesions, myelin proteolipid protein (PLP) staining pattern and density, as well as density of Olig2 ${ }^{+}$oligodendrocyte-lineage cells, were similar to the surrounding normal-appearing white matter. Our data from 25 iron $^{+}$ demyelinated and 14 iron $^{+}$remyelinated lesions show that both demyelinated and remyelinated lesions harbored similar levels of iron, but that levels of TfR and $\mathrm{HpC}$ were significantly higher in demyelinated lesions (Figure 5, A-D). In remyelinated lesions, oligodendrocyte-lineage cells harbored iron, whereas demyelinated lesions did not. Furthermore, demyelinated lesions harbored higher levels of TfR mRNA expression, but this expression was lost in remyelinated lesions (Figure 5E).

To further understand the significance of these changes in iron-regulating proteins, we used double immunohistochemistry to investigate their spatiotemporal variation throughout the course of lesion development (Figure 6). TfR in healthy white matter was found in $\mathrm{GFAP}^{+}$astrocytes and oligodendrocytelineage cells. However, as lesions became inflammatory and demyelinated (older than 2 weeks), oligodendrocyte-lineage cells were no longer detected. TfR levels in $\mathrm{Iba1}^{+}$microglia/macrophages increased, whereas levels decreased in astrocytes (Figure 6, A-D). In remyelinated lesions, by contrast, TfR was mainly found in astrocytes and oligodendrocyte-lineage cells, but not in microglia/ macrophages, similar to healthy white matter. In healthy white matter, FpN was found primarily in oligodendrocyte-lineage cells, and weakly in astrocytes and microglia/macrophages. Its level in astrocytes peaked when lesions were 2-6 weeks old, and it diminished in microglia/macrophages in 2- to 6-week-old and iron $^{+}$demyelinated lesions. HpC in the healthy white matter was present in all glial types studied, and as lesions evolved, its levels were highest in microglia/macrophages in 2- to 6-week-old and iron $^{+}$demyelinated lesions, but not in remyelinated lesions. These 
A
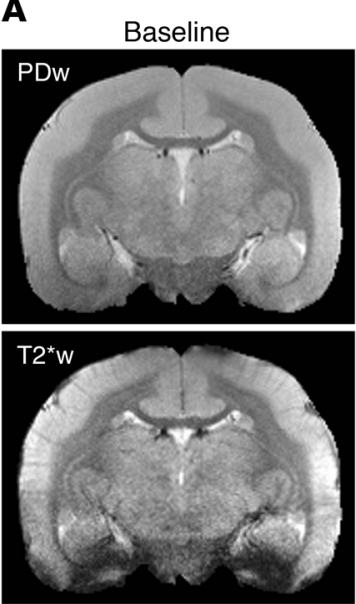

Lesion first detected
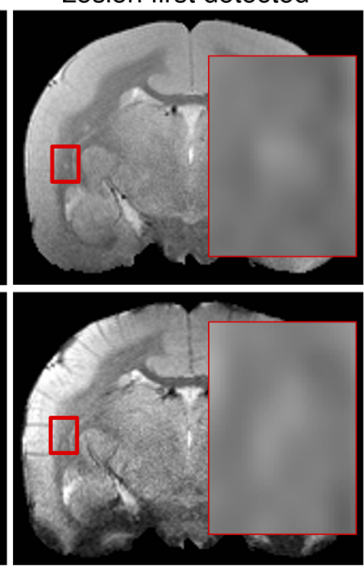

6-8 weeks after
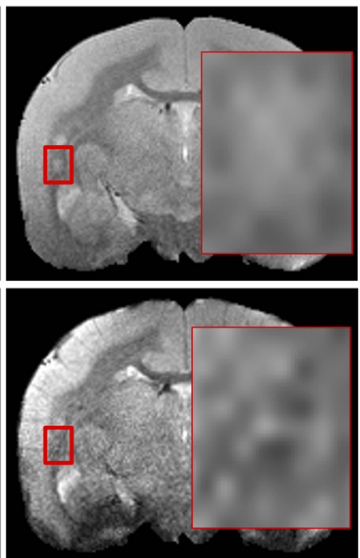
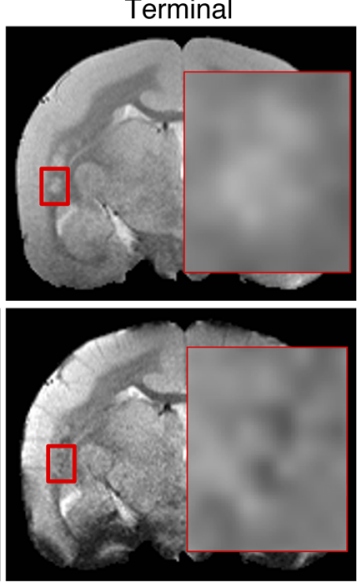

\section{B}
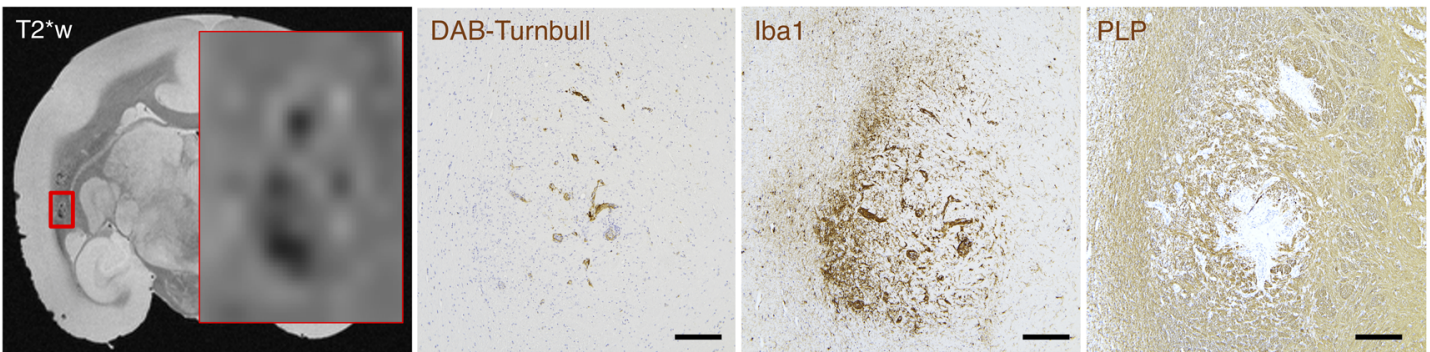

C

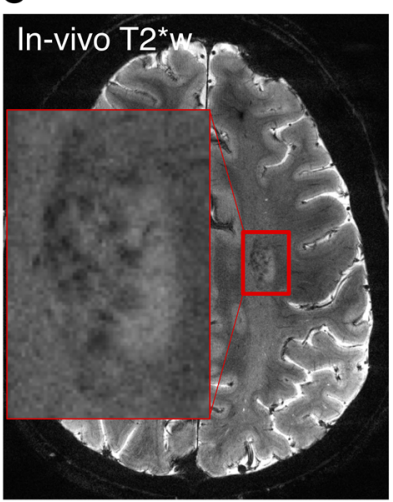

\section{D}
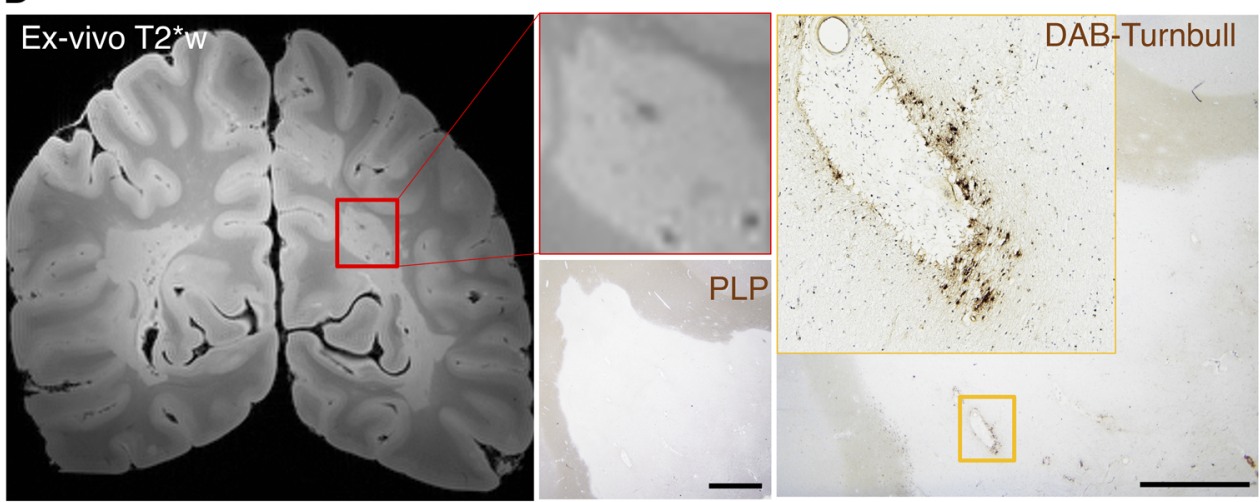

Figure 1. Iron accumulates in subacute and chronic (but not acute) marmoset EAE lesions, inside microglia/macrophages, and in human MS lesions. (A) In vivo MRI shows that once lesions are 6-8 weeks old, as determined by serial proton density-weighted (PDw) MRI (top row), they present a punctate hypointense signal on iron-sensitive T2*-weighted (T2* ${ }^{*}$ ) MRI (bottom row). (B) Intralesional hypointense signal on ex vivo T2* $\mathrm{W}$ MRI of the same lesion colocalizes with iron (DAB-Turnbull stain), as well as accumulation of microglia/macrophages (Iba1 immunohistochemistry) and demyelination (PLP immunohistochemistry). Note that the MRI section integrates over $100 \mu \mathrm{m}$ of tissue and overemphasizes the extent of iron due to the "blooming artifact," whereas the histopathological section is $5-\mu \mathrm{m}$ thick, potentially accounting for apparent detailed discrepancies between the 2 images. (C) In vivo T2* -weighted MRI detects punctate hypointensity, suggesting iron deposition, in a representative white matter lesion in MS (red box). (D) Ex vivo T2* W MRI also detects intralesional hypointense foci in MS (red box). Histopathology confirms the presence of demyelination (PLP), as well as perivascular iron deposition (DAB-Turnbull), in MS, in a similar spatial pattern as observed in the marmoset EAE lesions. Scale bars: $100 \mu \mathrm{m}$ for B, $20 \mu \mathrm{m}$ for D. Counterstain: hematoxylin. Representative lesion from marmoset 1. Red box indicates magnified view on interpolated MRI.

results show evidence of ongoing iron dysregulation in chronic, demyelinated lesions, whereas homeostasis is at least partially restored in remyelinated lesions, despite the presence of iron in both types of lesions.

We also investigated the potential involvement of HIF$2 \alpha$, a transcription factor that responds to iron deficiency and increases iron influx by promoting the production of DMT1 (44,
45). HIF- $2 \alpha$ is known to play an important role in regulation of iron homeostasis, including iron absorption $(46,47)$. Furthermore, HIF- $2 \alpha$ also has essential roles in the CNS, including in developmental myelination, and has been investigated for potential therapeutics in neurodegenerative disorders (48$50)$. In our lesions, however, we saw no temporal correlation between HIF-2 $\alpha$ levels and DMT1 (Supplemental Figure 3A). 
A

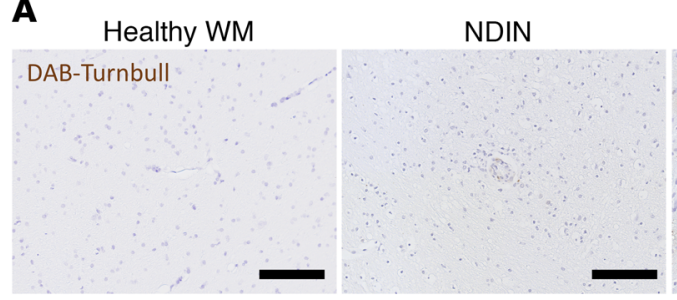

0-2 weeks old

2-6 weeks old

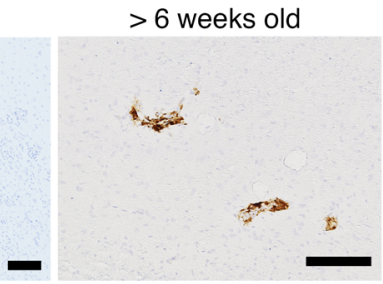

B
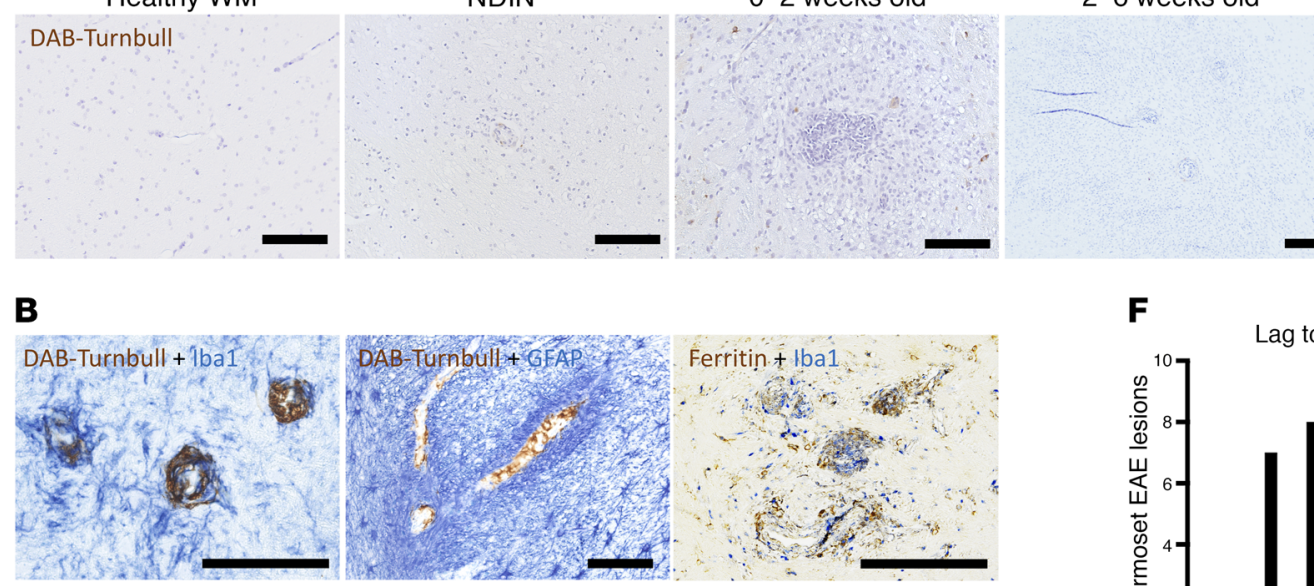

C

Iron ${ }^{+}$vs. ironmarmoset lesion age

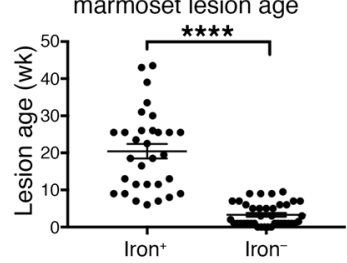

D

Iron $^{+/-}$lesions based on lesion age

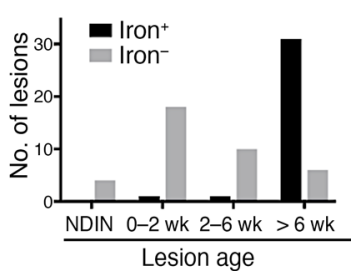

$\mathbf{E}$

Iron $^{+}$vs. iron ${ }^{-}$ lesion prevalence

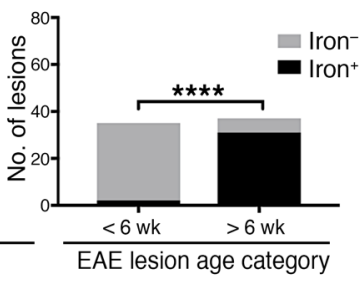

F

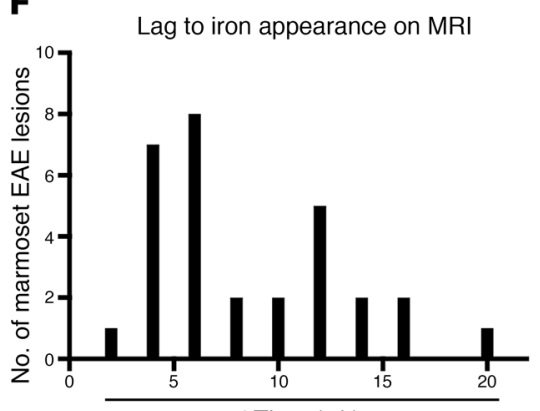

$\Delta$ Time (wk)

Figure 2. Iron is found inside microglia/macrophages in marmoset EAE lesions more than 6 weeks old. (A) Marmoset EAE lesions, categorized based on age, accumulate iron deposits only when they are older than 6 weeks; such deposits are not seen in healthy white matter (WM). (B) Double-staining in lesions older than 6 weeks shows that iron is found predominantly inside perivascular lba1+ microglia/macrophages, but not in GFAP ${ }^{+}$astrocytes. Ferritin and Iba1 double-staining shows localization of ferritin within Iba1+ cells in a similar distribution to the localization of iron. (C) Iron ${ }^{+}$lesions are significantly older than iron- lesions (Mann-Whitney test; distributions are summarized by mean and standard error). (D) Graphical representation of EAE lesions, categorized based on age and the presence or absence of intralesional iron. (E) Graphical representation of the proportion of lesions that are iron ${ }^{+}$or iron-, plotted as a function of lesion age (Fisher's exact test). (F) Histogram of the distribution of iron ${ }^{+}$lesions and the time difference between when the lesion was first detected on PDW and the hypointense signal was first detected on T2*W MRI. Scale bars: $100 \mu \mathrm{m}$. ${ }^{* * * *} P<0.0001$. Representative lesions from marmosets $9,10,12$, and 13.

HIF-2 $\alpha$ was mainly found inside oligodendrocytes (Supplemental Figure 3, B and C) but showed a temporal distribution pattern similar to that of any of the other iron regulators.

\section{Discussion}

Although dysregulation of iron has been associated with neurodegeneration and demyelination in MS, the inability to assess its dynamics in relation to mechanisms of tissue damage in vivo has impeded our understanding of its true role in disease pathogenesis. The fact that iron is essential for CNS myelinogenesis during development (2-4) raises the possibility that it may facilitate remyelination, even though most prior work in MS has focused on inflammation and neurodegeneration (5-9). MRI sequences, such as $\mathrm{T} 2^{*} \mathrm{~W}$ imaging, can be used in MS, especially at ultrahigh magnetic field ( 7 tesla), to characterize the spatiotemporal distribution of intralesional iron deposition; however, MRI can only provide limited pathobiological and temporal information in practical clinical studies. By using the marmoset EAE model, serial in vivo MRI, and histopathological analyses, we were able to assess the spatiotemporal dynamics of intralesional iron deposition, as well as the cellular and molecular composition of lesions before and after iron accumulation.
Our main descriptive result, gleaned from 13 marmosets with $\mathrm{EAE}$, is that iron is consistently found around small blood vessels in older ( $>6$ weeks) lesions. Importantly, we found that this iron deposition pattern can also be seen in human MS lesions, both in vivo and ex vivo, and is detectable on both MRI and histopathology. In the EAE lesions, iron appears to be bound to ferritin in microglia/macrophages, but it is not found in astrocytes.

To understand potential mechanisms leading to this celltype-specific iron accumulation, we investigated the ironregulating proteins $\mathrm{TfR}, \mathrm{FpN}$, and $\mathrm{HpC}$. We found that microglia/ macrophages had higher levels of TfR and $\mathrm{HpC}$, even before iron accumulation, particularly in lesions $0-2$ weeks old. This may simply be due to the fact that lesions at this stage have the highest density of microglia/macrophages (39). Increased TfR mRNA levels were also detected in these lesions, demonstrating local de novo production of TfR. Iron ${ }^{+}$demyelinated lesions older than 6 weeks also had similar characteristics. Either of these mechanisms (increase in iron influx or blockage of iron efflux) can lead to intracellular iron accumulation. Conversely, astrocytes had decreased levels of TfR but increased levels of FpN. Relevantly, this pattern of iron influx and efflux protein changes has been described in a rodent EAE study in the spinal cord (51). Our 
A

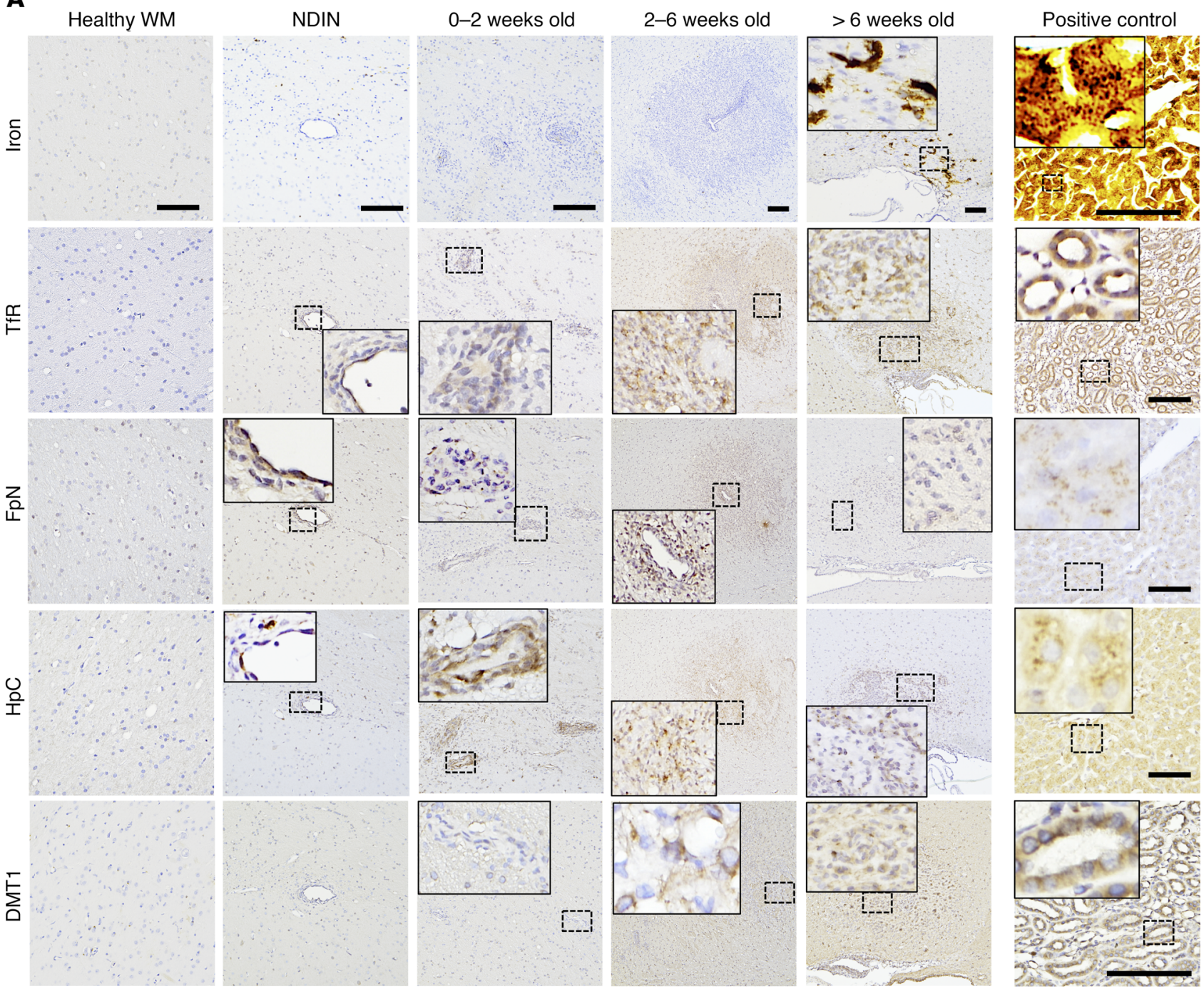

B

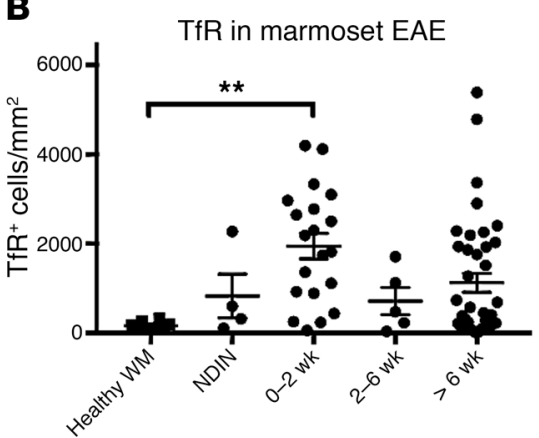

C

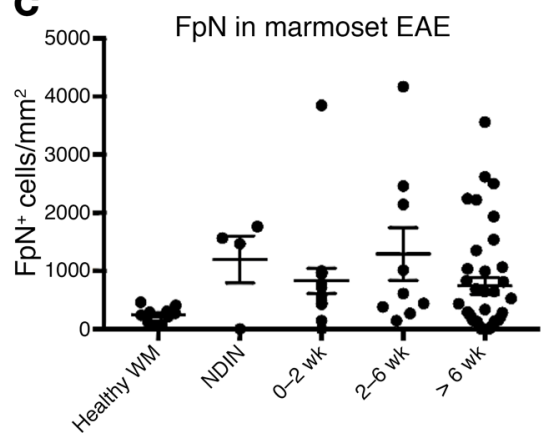

D

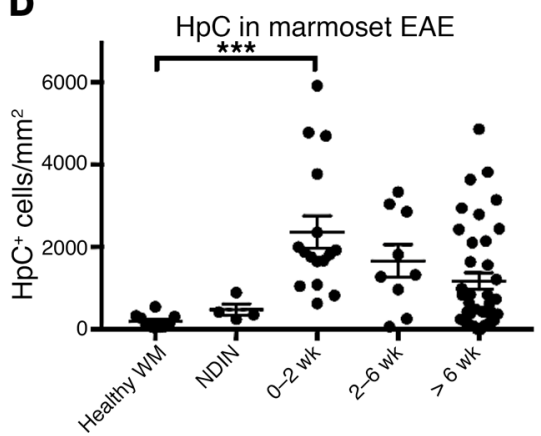

Figure 3. Iron-regulating protein levels change prior to iron accumulation. (A) Marmoset EAE lesions, categorized by age, and histopathology of iron and iron regulators, including transferrin receptor (TfR), ferroportin ( $F p N)$, hepcidin $(\mathrm{HpC})$, and divalent metal transporter 1 (DMT1). Staining of serial sections from representative lesions shows that the expression level of all of these proteins increased before the accumulation of iron (>6 weeks). (B-D) Quantification of TfR, FpN, and $\mathrm{HpC}$ expression level, with standard error of the mean. Dots represent individual lesions. Counterstain: hematoxylin. Scale bars: 100 $\mu \mathrm{m}$ and are constant within each column of the figure (except for the positive control). Positive controls: marmoset liver for iron, $\mathrm{FpN}$, and $\mathrm{HpC}$; marmoset kidney for TfR and DMT1. Lesions selected from marmosets 6, 7, 8, and 9. ${ }^{* *} P<0.01 ;{ }^{* *} P<0.001$ (ANOVA multiple comparisons test).

results in a nonhuman primate model of inflammatory demyelination corroborate the notion that time- and cell-type-specific shifts in iron regulation within the CNS influences intralesional iron accumulation.
We further investigated whether such time- and locationspecific iron deposition is pathogenic (i.e. proinflammatory), prorepair, or neither, by analyzing the expression levels of known markers of these processes in relation to temporal patterns of iron 


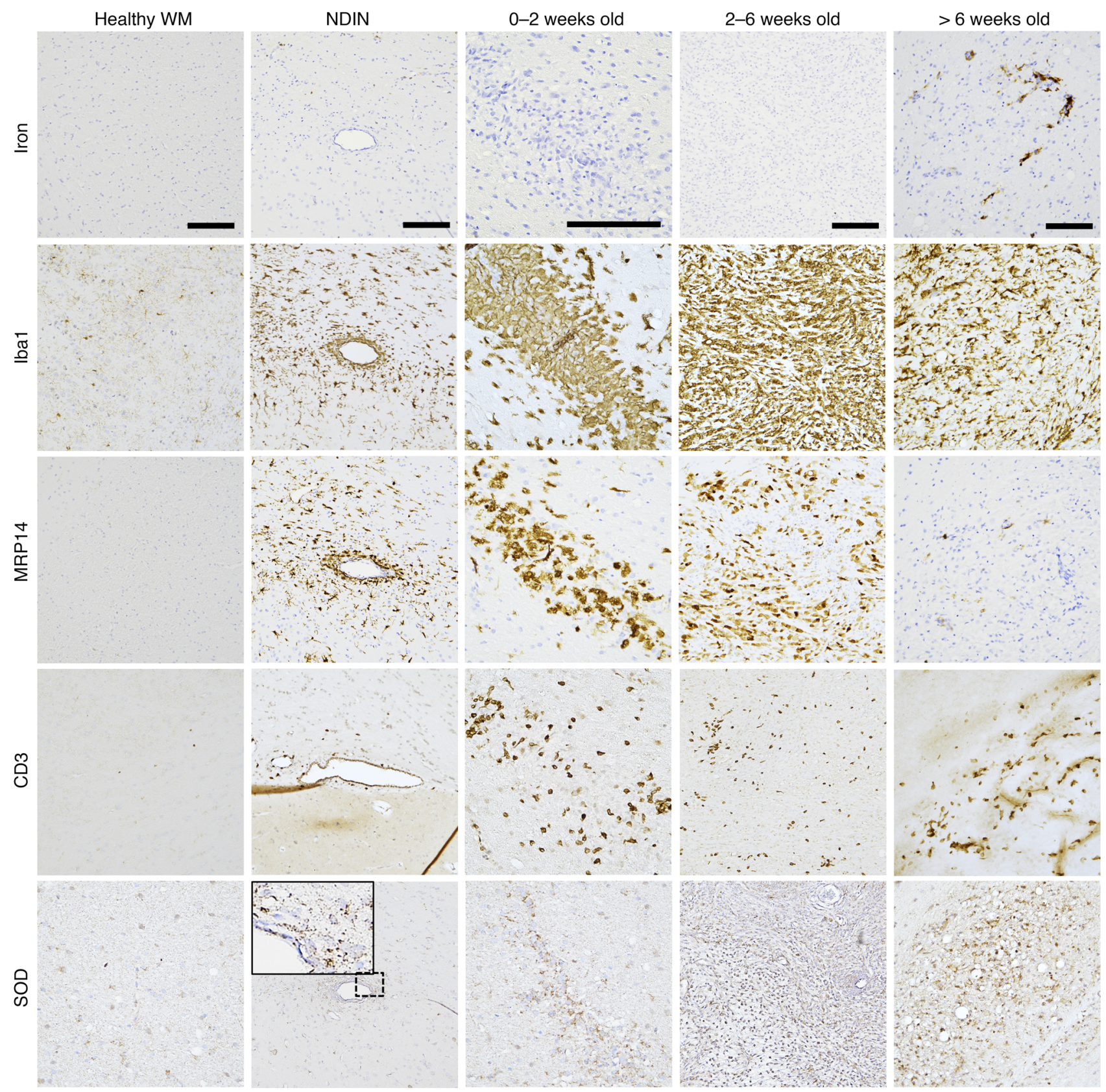

Figure 4. Iron accumulation is not associated with inflammation. Iba1+ microglia/macrophages, MRP14+ early-activated peripheral macrophages, and CD3 ${ }^{+}$ T cells, as well as superoxide dismutase (SOD, a marker for oxidative stress), are present from the earliest stage of lesion development (nondemyelinated inflammatory nodules [NDIN]), whereas iron accumulation only begins once lesions are 6 weeks old. Note that most Iba1+ microglia/macrophages do not contain iron, even in lesions older than 6 weeks. Scale bars: $100 \mu \mathrm{m}$. Counterstain: hematoxylin. Lesions selected from marmosets 6, 7, 9, and 10. Note that the lesion shown on the top row, second column, is the same lesion shown in Figure 3 A (top row, second column).

accumulation. Iron ${ }^{+}$microglia/macrophages can in principle cause inflammation and oxidative stress $(6,7,24,29)$. However, iron in our marmoset EAE lesions was stored in the form of ferritin inside microglia/macrophages, and ferritin-bound iron is highly stable, potentially even protective against iron-mediated toxicity through antioxidant mechanisms $(52,53)$. Furthermore, we found that inflammatory cells, including microglia/macrophages, MRP14 ${ }^{+}$ early-activated peripheral macrophages, and $\mathrm{T}$ cells, as well as superoxide dismutase (a marker of oxidative stress) all appeared within lesions much earlier than iron, i.e., during the first 2 weeks of lesion development. In prior work in mouse EAE, iron was also unassociated with early, destructive phases of the disease (51). In marmoset EAE, active demyelination occurred early in lesion development (39), whereas lesions older than 6 weeks showed signs of astrogliosis and extensive axonal damage and loss, without active demyelination. Taken together, these observations suggest that iron does not play an active role in the early, destructive phase of inflammatory demyelination.

To investigate whether iron may conversely be involved in the repair process, we categorized iron ${ }^{+}$lesions into either repaired/ 

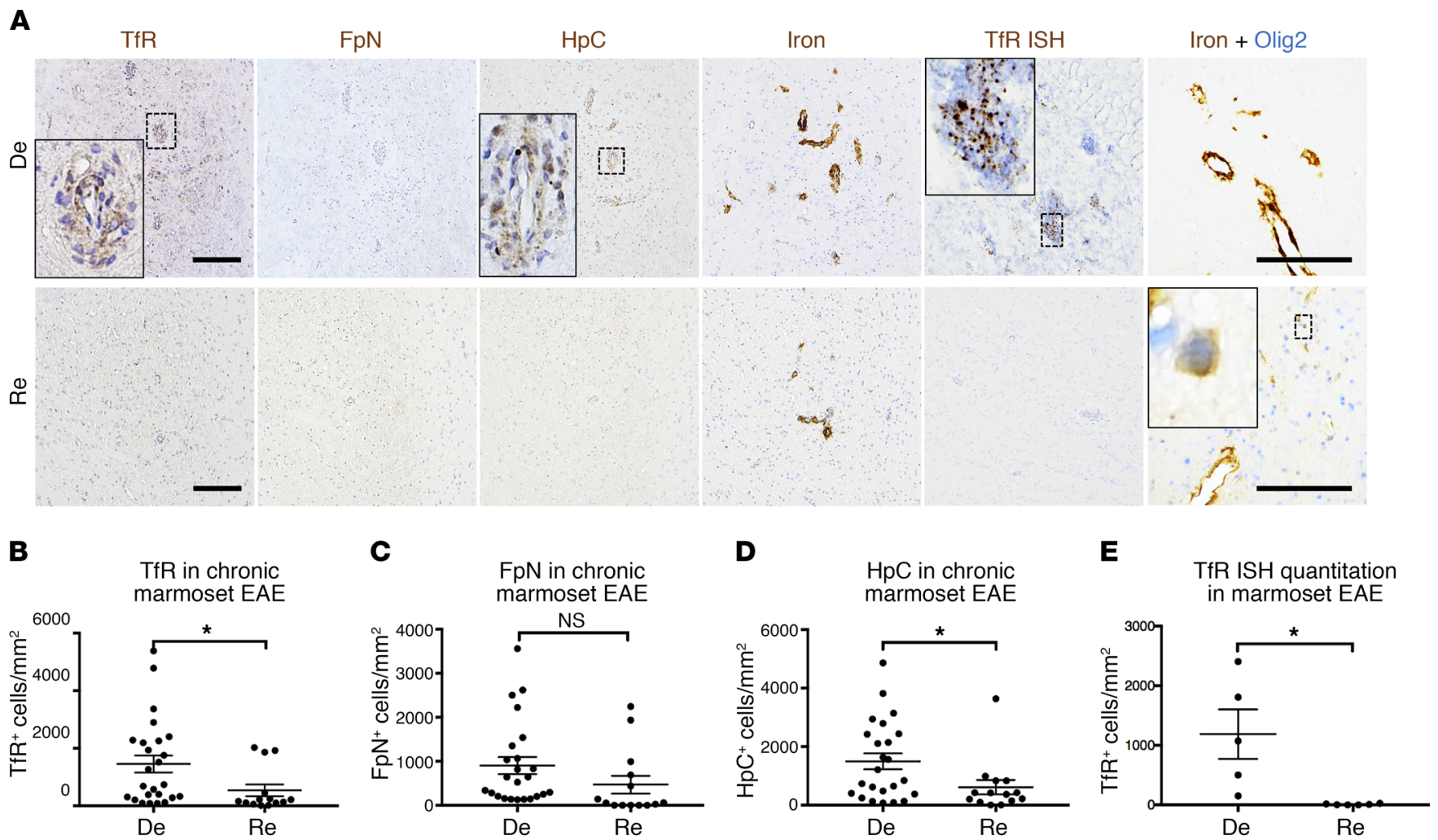

Figure 5. Remyelinated marmoset EAE lesions have lower levels of iron-regulating proteins than demyelinated lesions. (A) Immunohistochemical staining for representative demyelinated (De) and remyelinated (Re) chronic (>6 weeks old) EAE lesions show higher levels of transferrin receptor (TfR) and hepcidin $(\mathrm{HpC})$ in demyelinated compared with remyelinated lesions. However, both remyelinated and demyelinated lesions harbor iron. In remyelinated lesions, iron can be found inside Olig2+ oligodendrocyte-lineage cells. Quantification of (B) TfR, (C) ferroportin (FpN), (D) HpC, and (E) TfR mRNA shows that demyelinated lesions have higher numbers of TfR+ and $\mathrm{HpC}^{+}$cells per unit area, as well as higher TfR mRNA expression. Dots represent individual lesions. ${ }^{*} P<0.05$ (2-tailed $t$ test). Scale bars: $100 \mu \mathrm{m}$. Counterstain: hematoxylin for single-stained slides. Lesions selected from marmosets 1, 2, and 6 .

remyelinated or demyelinated lesions based on myelin protein staining, repopulation of oligodendrocyte-lineage cells, and in vivo PDw MRI. We found that demyelinated lesions had substantially higher levels of TfR and $\mathrm{HpC}$, whereas repaired lesions had levels similar to those in healthy white matter. Furthermore, whereas demyelinated lesions still showed elevated TfR mRNA expression, repaired lesions had significantly lower levels of TfR $\mathrm{mRNA}$. These findings suggest that chronically demyelinated iron $^{+}$lesions have ongoing iron dysregulation, specifically with respect to mechanisms that increase influx of iron into the lesion site.

Surprisingly, despite the differences in protein and mRNA expression levels of iron regulators between demyelinated and repaired lesions, both types of lesion harbored similar levels of intralesional iron. A possibility is that iron recruitment from the periphery occurs as part of the remyelination process, beginning with changes in iron-regulation proteins and culminating in iron entry into the lesion, where it is phagocytosed by iron-avid microglia/ macrophages that are a major part of the inflammatory reaction. Because iron is trapped within these cells due to downregulation of the efflux protein FpN, its levels may not reflect the actual degree of repair, as long as the microglia/macrophages themselves remain present. In the case of lesions that successfully remyelinate, iron-regulating protein levels return to prelesional state, consistent with the notion that iron recruitment is actively regulated. In fact, in remyelinated lesions, iron is found inside oligodendrocytelineage cells, and it remains possible that iron reaches these cells upon release from iron ${ }^{+}$microglia/macrophages.

Although mechanistic studies are difficult in the marmoset model due to lack of validated genetic and pharmacologic tools, we tested the hypothesis that iron accumulation and remyelination may be linked through the hypoxia inducible factor $2 \alpha$ (HIF-2 $\alpha$ ) pathway. HIF-2 $\alpha$ can increase in oligodendrocytes during myelination and remyelination (50). It is also upregulated in response to iron deficiency, thereby facilitating iron influx via increased production of DMT1 $(44,45)$. However, we did not observe a spatiotemporal appearance and distribution of HIF- $2 \alpha^{+}$cells that paralleled that of iron or iron-regulating protein levels, limiting our ability to make causal inferences. Our observation that HIF-2 $\alpha$ was expressed within oligodendrocyte-lineage cells, particularly in remyelinated lesions, is consistent with the general point that myelin synthesis and repopulation of iron ${ }^{+}$oligodendrocyte-lineage cells may go hand-in-hand with restoration of iron homeostasis, since appropriate redistribution and trafficking of iron from the periphery into the CNS is essential for developmental myelination $(9,54)$.

Overall, our results suggest a model in which iron is actively imported into the lesion environment in order to facilitate repair (Figure 7). In the context of inflammation, however, excess iron can become sequestered within iron-avid microglia/macrophages 
A

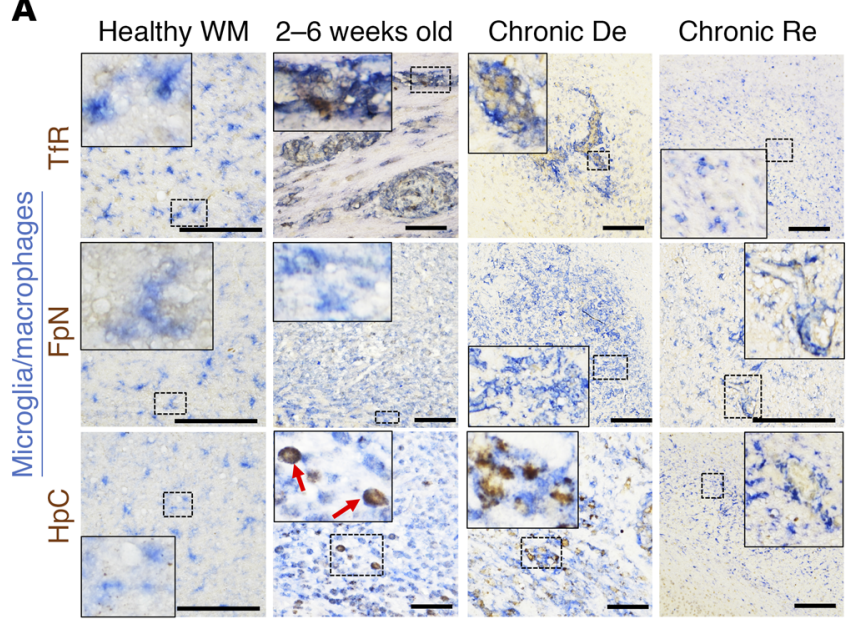

C

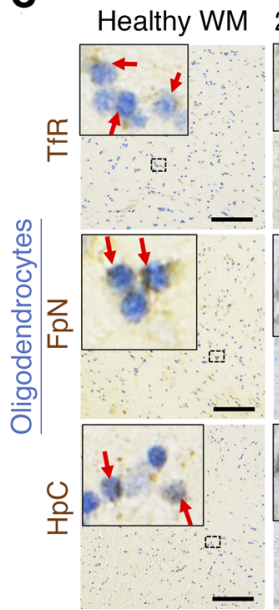

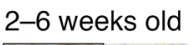

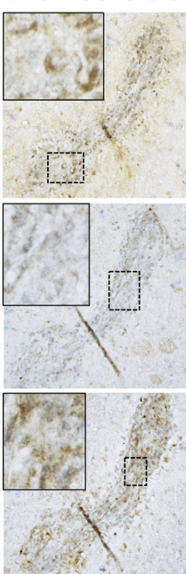

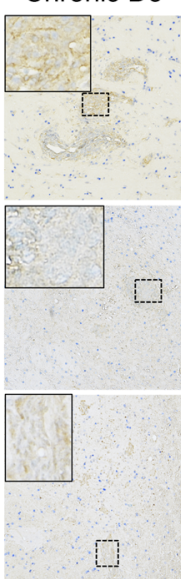

Chronic De

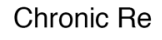

B
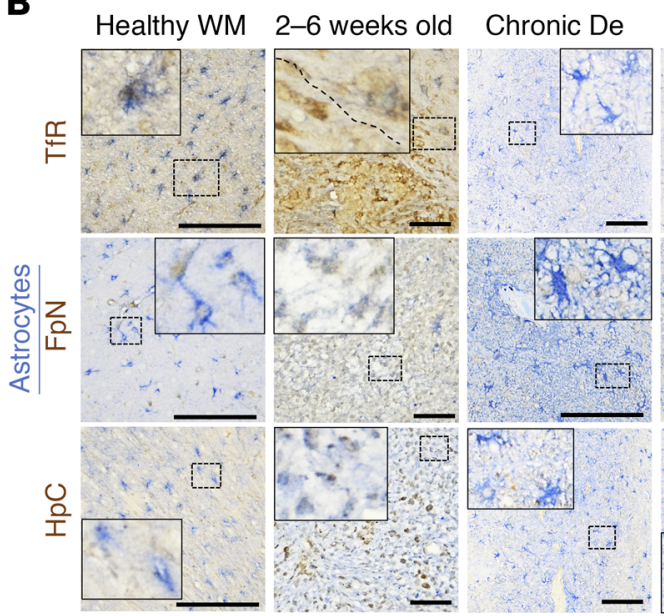

Chronic Re

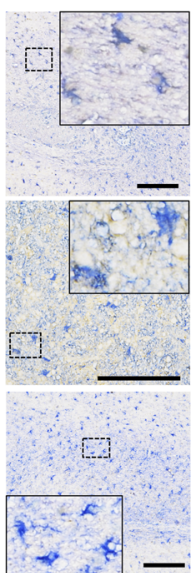

D
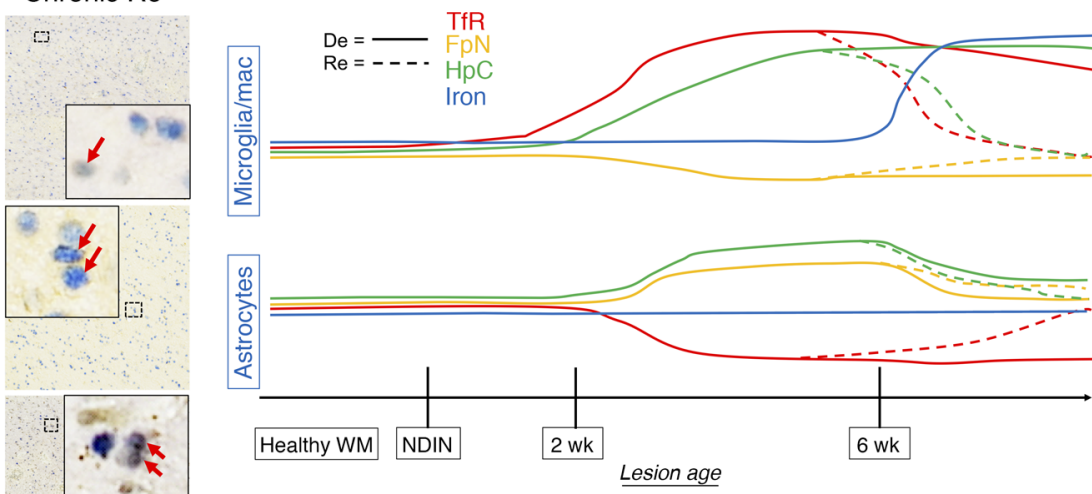

Figure 6. Iron-regulating protein changes are cell-specific and dynamic in healthy white matter and lesions. (A) Panel of double stains (Iba1 for microglia/ macrophages, together with the iron-regulating proteins transferrin receptor (TfR), ferroportin ( $\mathrm{FpN}$ ), and hepcidin (HpC)) in healthy white matter and various stages of marmoset EAE lesions. Microglia/macrophages weakly express all 3 iron-regulating proteins in healthy white matter. As lesions age, TfR and HpC levels increase, remaining high in chronically demyelinated (De) lesions but returning to baseline in remyelinated (Re) lesions. On the other hand, FpN levels slightly drop during demyelination but also return to normal upon remyelination. (B) Panel of double stains (GFAP for astrocytes, together with the same iron-regulating proteins). In the healthy white matter, astrocytes express TfR, which is lost upon demyelination but returns with remyelination. FpN and $\mathrm{HpC}$ levels increase in 2- to 6-week-old and chronically demyelinated lesions but also return to baseline with remyelination. (C) Panel of double stains (Olig2 for oligodendrocyte-lineage cells, likely a mixture of oligodendrocyte precursor cells and mature oligodendrocytes, together with iron-regulating proteins). In healthy white matter, TfR, $\mathrm{FpN}$, and $\mathrm{HpC}$ are all expressed in the oligodendrocyte lineage (red arrows). In 2- to 6-week-old and chronically demyelinated lesions, oligodendrocyte-lineage cells are not detected. In repaired/remyelinated lesions, repopulated oligodendrocyte-lineage cells show all 3 proteins at relatively normal levels (red arrows). (D) Summary of iron regulation changes in microglia/macrophages and astrocytes during marmoset EAE lesion development and repair. Scale bars: $100 \mu \mathrm{m}$. Lesions selected from marmosets 1, 2, 6, 8, and 10.

and thereby remain within lesions even after they remyelinate. In this model, iron is necessary for repair, but not sufficient. This begs the question of the source of iron in these lesions, with possibilities that include lysed oligodendrocytes, myelin debris, red blood cells, and transport from outside the CNS in transferrin-bound form. The first 2 possibilities are less likely given the relative timing of demyelination and oligodendrocyte death in these lesions, which occur weeks before iron accumulation. Further, we found no signs of hemorrhage in the lesions, suggesting that iron does not derive from released hemosiderin of lysed red blood cells. However, we found that as lesions aged, the expression levels of TfR in the endothelium within the lesions increased, suggesting that iron may enter lesions from the periphery in transferrin- bound form. TfR in the brain capillary endothelium is known to maintain transferrin-bound iron transport into the parenchyma (55-58). Although the mechanism by which iron influx is signaled remains unclear, there are numerous examples of links between inflammation and iron changes. For example, in anemia of chronic disease, increased production of $\mathrm{HpC}$ can lead to intracellular iron accumulation (59-61).

In addition to limited ability to manipulate iron levels experimentally, a caveat of the current study is that some marmosets were inoculated with herpesvirus together with human white matter homogenate. Such multiple use of primates is not uncommon, given that they are a scarce resource. However, in this specific context, and even though HHV6 can affect the clinical course of 


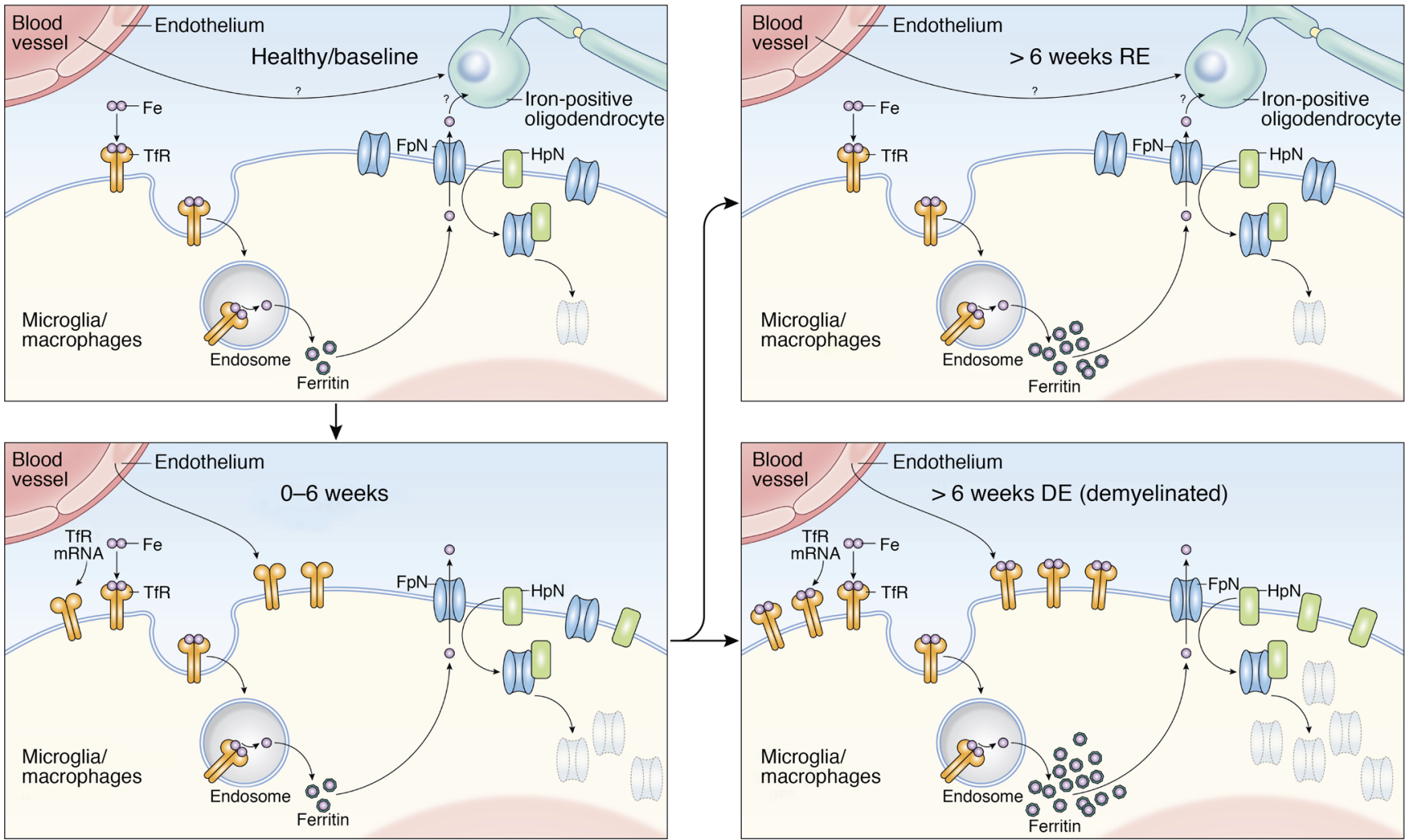

Figure 7. Proposed model to explain perivascular iron deposition inside microglia/macrophages in marmoset EAE lesions. Iron accumulates inside microglia/macrophages, which show increased expression of transferrin receptor (TfR), a main iron influx protein. Iron itself is transported across the endothelium of nearby blood vessels, which also upregulates TfR. Increased levels of hepcidin ( $\mathrm{HpC}$ ), which binds, internalizes, and degrades ferroportin $(\mathrm{FpN})$, the main iron efflux channel protein, also contributes to intracellular iron accumulation. Iron regulation returns to homeostasis in remyelinated lesions, though iron levels within these cells remain high. Demyelinated lesions continue to show both dysregulated iron metabolism and persistent iron deposition. The source of iron observed in the oligodendrocyte-lineage cells of remyelinated lesions is not addressed by this study, but possible mechanisms include both direct transendothelial transport and release from microglia/macrophages.

EAE (62), we did not observe any difference with respect to iron accumulation and dysregulation in our study - nor did we observe such differences according to marmoset age or dose of the white matter homogenate used for EAE induction.

In conclusion, intracellular iron deposition shows a specific spatial and temporal deposition inside marmoset EAE lesions. Similarly, in a cohort of 39 MS cases, more than half had at least 1 iron-containing lesion, suggesting that the perivascular iron deposition detected in marmoset EAE lesions is a characteristic and relevant pathobiological phenomenon in MS pathophysiology. This pattern of iron deposition is distinct from the previously described rim-like deposition, where iron is found at the edge of chronically demyelinating MS lesions in association with smoldering inflammation and ongoing demyelination and lesion expansion $(11,18,63)$.

Our time course and gene expression data suggest a potential role for iron in repair/remyelination rather than inflammatory demyelination per se, with iron most likely being shuttled from the periphery via TfR in the CNS vascular endothelium. In order to further elucidate the precise role of intralesional iron deposition, future in vitro and in vivo studies should investigate whether manipulating the signaling pathways involved in iron accumulation interferes with lesion repair.

\section{Methods}

Marmoset EAE induction. The current study involved 13 marmosets (5 males and 8 females, ages 2-6 years at baseline) (Supplemental Table 1). As primates are a rare resource, animals were pooled from several different studies. Nine of the 13 marmosets were injected with $600 \mu \mathrm{L}$ white matter homogenate, mixed in complete Freund's adjuvant (Difco Laboratories) as described previously (39). The injection included 200 mg human white matter mixed with minimal PBS, $250 \mu \mathrm{L}$ incomplete Freund's adjuvant (Difco Laboratories), and $1.8 \mathrm{mg}$ desiccated $\mathrm{Myco}$ bacterium tuberculosis (Difco Laboratories). Some marmosets from the group were also involved in another investigation, and some were coimmunized with human herpes virus (HHV) intranasally (64). The remaining 4 marmosets were first injected with a half-dose of human white matter homogenate $(100 \mathrm{mg})$. When no signs of lesion formation were detected 2 months after injection, they were injected with an additional full-dose of human white matter homogenate $(200 \mathrm{mg})$. All protocols were approved by the National Institute of Neurological Disorders and Stroke Animal Care and Use Committee.

Marmoset in vivo MRI. Marmosets were regularly scanned on a weekly or a biweekly basis under anesthesia, as previously described (65). To identify lesion formation, we used PDw, which is sensitive to changes over time as lesions evolve (66) (Figure 1A). To identify iron deposition, we used $\mathrm{T} 2{ }^{*} \mathrm{~W}$ MRI. Sequence parameters are described 
in Supplemental Table 2. Imaging parameters were similar but not identical across marmosets.

Human in vivo and ex vivo MRI. After observing suspected intralesional iron depositions in MS patients from cross-sectional in vivo MRI, we ran a pilot analysis to test the prevalence of iron ${ }^{+}$MS lesions. In vivo brain scans in 39 consecutively studied participants in an IRB-approved MS natural history protocol, studied on a 7 tesla wholebody research system (Siemens), were analyzed. MS lesions and iron deposition were detected using $\mathrm{T} 2{ }^{*} \mathrm{~W}$ MRI. Specific sequence parameters are described in Supplemental Table 2.

For ex vivo MRI, 4 donated brains from individuals with MS were used to identify intralesional iron depositions. In total, 15 lesions were identified and analyzed as previously described (67), specifically for iron deposition using both T2* ${ }^{*}$ MRI (sequence parameters in Supplemental Table 2) and histopathology. Demographics are described in Supplemental Table 3.

Histopathology. Following necropsy immediately after death, marmoset brains were collected and fixed in $4 \%$ paraformaldehyde. Customized 3D-printed brain cradles were used for lesion identification, as previously described $(68,69)$. Human brains were collected at autopsy and fixed in $10 \%$ neutral-buffer formalin. Both marmoset and human brain slabs were embedded in paraffin, cut into 4- to $10-\mu \mathrm{m}$-thick slides, and placed on SuperFrost Plus (Thermo Fisher Scientific) slides. All the slides went through routine deparaffinization using xylene and serial rehydration.

To visualize iron deposition, diaminobenzidine-enhanced (DABenhanced) Turnbull staining was performed, where slides were immersed in 2\% ammonium sulfide solution for 90 minutes, incubated with $10 \%$ potassium ferricyanide and $0.5 \%$ hydrochloric acid solution for 15 minutes in $37^{\circ} \mathrm{C}$, and incubated with $0.3 \%$ hydrogen peroxide in methyl alcohol solution for 1 hour. The slides then were developed using DAB (Abcam) and counterstained using hematoxylin (Leica Biosystems Inc.).

For immunohistochemistry, either the immunoperoxidase or the alkaline phosphatase (AP) method was used. For each marker of interest, slides went through the respective antigen retrieval, protein blocking, and primary and secondary antibody inoculations (Supplemental Table 4). Slides developed using the immunoperoxidase method were developed with $\mathrm{DAB}$, counterstained with hematoxylin, and differentiated with Blue Buffer (Leica Biosystems Inc.). Slides going through double-staining (i.e. ferritin and Iba1) were stained first using the immunoperoxidase method followed by AP Vector Blue (Vector Laboratories), without counterstaining.

Histopathological lesion characterization and quantification. Lesions identified on histopathology were retrospectively referenced back to in vivo MRI, and were categorized based on age into the following groups: NDINs; 0- to 2-week-old lesions; 2- to 6-week-old lesions; demyelinated lesions older than 6 weeks; or remyelinated lesions older than 6 weeks. NDINs are the earliest stage of lesion development and are seen on histopathology but not in vivo structural MRI, as previously described $(21,39)$. Repaired (likely remyelinated) lesions were categorized based on qualitative assessment of in vivo PDW MRI (assessed on the basis of whether the originally hyperintense lesion returned to isointensity with extralesional white matter), immunohistochemical analysis of myelin (PLP), and repopulation of oligodendrocytes. Prior work from our group shows that EAE lesions that show signal intensity changes on PDw MRI are invariably demyelinated (as confirmed by postmortem histopathology) $(21,39)$. Thus, lesions that are originally hyperintense and return to isointensity, and that demonstrate normal myelin and oligodendrocyte characteristics, can be characterized as radiologically remyelinated. For lesions without clear lesion borders on histopathology, or that underwent dynamic changes on serial in vivo MRI, we defined the lesion based on the estimated size at peak on in vivo MRI and corrected for in vivo-to-postmortem changes.

Quantification for the area density of cells was performed using Fiji ImageJ software, as previously described (39). All the lesions and slides were carefully interpreted and score by a blinded, boardcertified, veterinary pathologist. Histopathological images were taken using an Observer 1 microscope (Zeiss), an Eclipse Ci-L LED Microscope, and a DSRi2 Color Digital Camera (Nikon Instruments).

In situ hybridization of transferrin receptor. In situ hybridization (ISH) protocol was performed as recommended in the RNAscope $2.5 \mathrm{HD}$ Chromogenic Assay user manual. Briefly, sections were dried onto slides $\left(60^{\circ} \mathrm{C}\right)$, deparaffinized, rehydrated, and incubated with $3 \%$ hydrogen peroxide for 10 minutes. The sections were then boiled $\left(99^{\circ} \mathrm{C}-104^{\circ} \mathrm{C}\right)$ in the Target Retrieval solution (Advanced Cell Diagnostics [ACD]) and treated with protease for 30 minutes at $40^{\circ} \mathrm{C}$. TfR mRNA probe was designed with ACD (target: marmoset transferrin receptor [TRFC, NM_001301847.1, target region 2-1036, catalog 538131]). Tissue sections were incubated with the TfR probe for 2 hours at $40^{\circ} \mathrm{C}$ using ACD HybEZ ii Hybridization System (https://acdbio.com/hybez-ii-system). After application of washing buffer, sections were incubated with Amp1 to Amp6 (6 consecutive steps) for $15-30$ minutes each at $40^{\circ} \mathrm{C}$ using HybEZä II Hybridization System. Final ISH signal was detected using DAB solution for 10 minutes at room temperature, and slides were then counterstained with hematoxylin.

Transmission electron microscopy. In a subset of marmosets, demyelinated and remyelinated lesions were examined and compared at the ultrastructural level by transmission electron microscopy (TEM). Briefly, tissues were perfused with $4 \%$ paraformaldehyde, fixed with $10 \%$ formalin, and again with $20 \mathrm{~mL}$ PBS containing $2 \%$ paraformaldehyde and $2.5 \%$ glutaraldehyde (Electron Microscopy Sciences), and postfixed at $4^{\circ} \mathrm{C}$ for 3 days. Vibratome sections $(100 \mu \mathrm{m})$ were prepared and stained with $1 \%$ osmium tetroxide. Cut slides were dehydrated through graded ethanol and embedded in epoxy resin. The semithin sections were prepared by Leica Ultracut E Ultramicrotome, and were stained with toluidine blue solution. Once the lesions were identified by light microscopy, ultrathin sections were examined with a transmission electron microscope (JEOL 1200 EXII Microscope).

Statistics. GraphPad Prism 7 software was used for all statistical analyses. For parametric data analysis, ordinary 1-way analysis of variance (ANOVA) was used for comparing lesions. All graphic data (scatter plots) represent mean and standard error of the mean. For comparing demyelinated versus remyelinated lesions, we used the unpaired 2 -tailed $t$ test. To compare the proportion of iron ${ }^{+}$and iron ${ }^{-}$lesions in young ( $<6$ weeks old) and old ( $>6$ weeks old) lesions, Fisher's exact test was used. The following statistical significances were used for all the analyses: ${ }^{*} P<0.05 ;{ }^{* *} P<0.01 ;{ }^{* * *} P<0.001 ;{ }^{* * *} P<0.0001$.

\section{Author contributions}

NJ Lee and DSR wrote the manuscript. NJ Lee and SKH performed histopathological staining and statistical analyses. NJ Lee, PS, MA, GN, NJ Luciano, ECL, and CCY performed human data collection and/or animal experiments. NJ Lee, TAR, ACS, SJ, 
and DSR helped with the conception of experimental ideas and scientific questions. All authors critically reviewed the manuscript for intellectual content and approved the final version.

\section{Acknowledgments}

We thank the National Institute of Neurological Disorders and Stroke (NINDS) Neuroimmunology Clinic and the NIH Functional Magnetic Resonance Imaging Facility for coordinating the acquisition of MRI scans, Mahin S. Hossain for helping with data analysis, and the NIH Medical Arts Branch for the artwork. We also thank Susan Cheng from the NINDS Elec- tron Microscopy Facility for helping with tissue preparation and image acquisition. This research was supported by the Intramural Research Program of NINDS and the Adelson Medical Research Foundation.

Address correspondence to: Daniel S. Reich, Building 10, Room 5C103, 10 Center Drive MSC 1400, Bethesda, Maryland 20892, USA. Phone: 301.496.1801; Email: daniel.reich@nih.gov.

ACS's present address is: Department of Neurobiology, University of Pittsburgh Brain Institute, Pittsburgh, Pennsylvania, USA.
1. Reich DS, Lucchinetti CF, Calabresi PA. Multiple sclerosis. N Engl J Med. 2018;378(2):169-180.

2. Connor JR, Menzies SL. Cellular management of iron in the brain. J Neurol Sci.1995;134 Suppl:33-44.

3. Connor JR, Menzies SL. Relationship of iron to oligodendrocytes and myelination. Glia. 1996;17(2):83-93.

4. Todorich B, Pasquini JM, Garcia CI, Paez PM, Connor JR. Oligodendrocytes and myelination: the role of iron. Glia. 2009;57(5):467-478.

5. Hametner S, Wimmer I, Haider L, Pfeifenbring S, Brück W, Lassmann H. Iron and neurodegeneration in the multiple sclerosis brain. Ann Neurol. 2013;74(6):848-861.

6. Mehta V, et al. Iron is a sensitive biomarker for inflammation in multiple sclerosis lesions. PLOS ONE. 2013;8(3):e57573.

7. Thomsen MS, Andersen MV, Christoffersen PR, Jensen MD, Lichota J, Moos T. Neurodegeneration with inflammation is accompanied by accumulation of iron and ferritin in microglia and neurons. Neurobiol Dis. 2015;81:108-118.

8. Adamczyk B, Adamczyk-Sowa M. New insights into the role of oxidative stress mechanisms in the pathophysiology and treatment of multiple sclerosis. Oxid Med Cell Longev. 2016;2016:1973834.

9. Stephenson E, Nathoo N, Mahjoub Y, Dunn JF, Yong VW. Iron in multiple sclerosis: roles in neurodegeneration and repair. Nat Rev Neurol. 2014;10(8):459-468.

10. Bagnato F, et al. Tracking iron in multiple sclerosis: a combined imaging and histopathological study at 7 Tesla. Brain. 2011;134 (Pt 12):3602-3615.

11. Dal-Bianco A, et al. Slow expansion of multiple sclerosis iron rim lesions: pathology and $7 \mathrm{~T}$ magnetic resonance imaging. Acta Neuropathol. 2017;133(1):25-42.

12. Bozin I, et al. Magnetic resonance phase alterations in multiple sclerosis patients with short and long disease duration. PLOS ONE. 2015;10(7):e0128386.

13. Chawla S, et al. Iron and non-iron-related characteristics of multiple sclerosis and neuromyelitis optica lesions at 7T MRI. AJNR Am J Neuroradiol. 2016;37(7):1223-1230.

14. Haacke EM, et al. Characterizing iron deposition in multiple sclerosis lesions using susceptibility weighted imaging. J Magn Reson Imaging. 2009;29(3):537-544.

15. Hagemeier J, et al. Iron deposition in multiple sclerosis lesions measured by susceptibili- ty-weighted imaging filtered phase: a case control study. J Magn Reson Imaging. 2012;36(1):73-83.

16. Zhang $Y$, et al. Longitudinal change in magnetic susceptibility of new enhanced multiple sclerosis (MS) lesions measured on serial quantitative susceptibility mapping (QSM). JMagn Reson Imaging. 2016;44(2):426-432.

17. Zhang Y, et al. Quantitative susceptibility mapping and $\mathrm{R} 2^{*}$ measured changes during white matter lesion development in multiple sclerosis: myelin breakdown, myelin debris degradation and removal, and iron accumulation. AJNR Am J Neuroradiol. 2016;37(9):1629-1635.

18. Absinta M, et al. Persistent 7-tesla phase rim predicts poor outcome in new multiple sclerosis patient lesions. J Clin Invest. 2016;126(7):2597-2609.

19. 't Hart BA, Massacesi L. Clinical, pathological, and immunologic aspects of the multiple sclerosis model in common marmosets (Callithrix jacchus). J Neuropathol Exp Neurol. 2009;68(4):341-355.

20. Kap YS, Laman JD, 't Hart BA. Experimental autoimmune encephalomyelitis in the common marmoset, a bridge between rodent EAE and multiple sclerosis for immunotherapy development. J Neuroimmune Pharmacol. 2010;5(2):220-230.

21. Maggi $P$, et al. The formation of inflammatory demyelinated lesions in cerebral white matter. Ann Neurol. 2014;76(4):594-608.

22. Maggi P, Sati P, Massacesi L. Magnetic resonance imaging of experimental autoimmune encephalomyelitis in the common marmoset. J Neuroimmunol. 2017;304:86-92.

23. Dunham J, et al. Oxidative injury and iron redistribution are pathological hallmarks of marmoset experimental autoimmune encephalomyelitis. J Neuropathol Exp Neurol. 2017;76(6):467-478.

24. Mairuae N, Connor JR, Cheepsunthorn P. Increased cellular iron levels affect matrix metalloproteinase expression and phagocytosis in activated microglia. Neurosci Lett. 2011;500(1):36-40.

25. Kroner A, Greenhalgh AD, Zarruk JG, Passos Dos Santos R, Gaestel M, David S. TNF and increased intracellular iron alter macrophage polarization to a detrimental M1 phenotype in the injured spinal cord. Neuron. 2014;83(5):1098-1116.

26. Lampron A, et al. Inefficient clearance of myelin debris by microglia impairs remyelinating processes. J Exp Med. 2015;212(4):481-495.

27. Bresgen N, Eckl PM. Oxidative stress and the homeodynamics of iron metabolism. Biomolecules. 2015;5(2):808-847.

28. Ghosh MC, Zhang DL, Rouault TA. Iron misreg- ulation and neurodegenerative disease in mouse models that lack iron regulatory proteins. Neurobiol Dis. 2015;81:66-75.

29. Haider L. Inflammation, Iron, Energy Failure, and Oxidative Stress in the Pathogenesis of Multiple Sclerosis. Oxid Med Cell Longev. 2015;2015:725370

30. Mills E, Dong XP, Wang F, Xu H. Mechanisms of brain iron transport: insight into neurodegeneration and CNS disorders. Future Med Chem. 2010;2(1):51-64.

31. Xie Y, et al. Ferroptosis: process and function. Cell Death Differ. 2016;23(3):369-379.

32. Lynch SG, Peters K, LeVine SM. Desferrioxamine in chronic progressive multiple sclerosis: a pilot study. Mult Scler. 1996;2(3):157-160.

33. Lynch SG, Fonseca T, LeVine SM. A multiple course trial of desferrioxamine in chronic progressive multiple sclerosis. Cell Mol Biol (Noisyle-grand). 2000;46(4):865-869.

34. Norstrand IF, Craelius W. A trial of deferoxamine (Desferal $^{*}$ ) in the treatment of multiple sclerosis. A pilot study. Clinical Trials J.1989;26(6):365-369.

35. Bowern N, Ramshaw IA, Clark IA, Doherty PC. Inhibition of autoimmune neuropathological process by treatment with an iron-chelating agent. J Exp Med. 1984;160(5):1532-1543.

36. Pedchenko TV, LeVine SM. Desferrioxamine suppresses experimental allergic encephalomyelitis induced by MBP in SJL mice. J Neuroimmunol. 1998;84(2):188-197.

37. Willenborg DO, Bowern NA, Danta G, Doherty PC. Inhibition of allergic encephalomyelitis by the iron chelating agent desferrioxamine: differential effect depending on type of sensitizing encephalitogen. JNeuroimmunol.1988;17(2):127-135.

38. Guy J, McGorray S, Qi X, Fitzsimmons J, Mancuso A, Rao N. Conjugated deferoxamine reduces blood-brain barrier disruption in experimental optic neuritis. Ophthalmic Res. 1994;26(5):310-323.

39. Lee NJ, et al. Spatiotemporal distribution of fibrinogen in marmoset and human inflammatory demyelination. Brain. 2018;141(6):1637-1649.

40. Badaracco ME, Siri MV, Pasquini JM. Oligodendrogenesis: the role of iron. Biofactors. 2010;36(2):98-102.

41. Moos T, Rosengren Nielsen T, Skjørringe T, Morgan EH. Iron trafficking inside the brain J Neurochem. 2007;103(5):1730-1740.

42. Morath DJ, Mayer-Pröschel M. Iron modulates the differentiation of a distinct population of glial precursor cells into oligodendrocytes. Dev Biol. 2001;237(1):232-243. 
43. Schonberg DL, McTigue DM. Iron is essential for oligodendrocyte genesis following intraspinal macrophage activation. Exp Neurol. 2009;218(1):64-74.

44. Anderson ER, et al. Intestinal HIF2 $\alpha$ promotes tissue-iron accumulation in disorders of iron overload with anemia. Proc Natl Acad Sci U S A. 2013;110(50):E4922-E4930.

45. Mastrogiannaki M, Matak P, Keith B, Simon MC, Vaulont S, Peyssonnaux C. HIF-2alpha, but not HIF-1alpha, promotes iron absorption in mice. J Clin Invest. 2009;119(5):1159-1166.

46. Peyssonnaux C, et al. Regulation of iron homeostasis by the hypoxia-inducible transcription factors (HIFs). J Clin Invest. 2007;117(7):1926-1932.

47. Taylor M, et al. Hypoxia-inducible factor- $2 \alpha$ mediates the adaptive increase of intestinal ferroportin during iron deficiency in mice. Gastroenterology. 2011;140(7):2044-2055.

48. Ashok BS, Ajith TA, Sivanesan S. Hypoxiainducible factors as neuroprotective agent in Alzheimer's disease. Clin Exp Pharmacol Physiol. 2017;44(3):327-334.

49. Merelli A, Rodríguez JCG, Folch J, Regueiro MR, Camins A, Lazarowski A. Understanding the role of hypoxia inducible factor during neurodegeneration for new therapeutics opportunities. Curr Neuropharmacol. 2018;16(10):1484-1498.

50. Yuen TJ, et al. Oligodendrocyte-encoded HIF function couples postnatal myelination and white matter angiogenesis. Cell. 2014;158(2):383-396.

51. Zarruk JG, et al. Expression of iron homeostasis proteins in the spinal cord in experimental autoimmune encephalomyelitis and their implications for iron accumulation. Neurobiol Dis.
2015;81:93-107.

52. Arosio P, Levi S. Cytosolic and mitochondrial ferritins in the regulation of cellular iron homeostasis and oxidative damage. Biochim Biophys Acta. 2010;1800(8):783-792.

53. Theil EC. Ferritin: the protein nanocage and iron biomineral in health and in disease. Inorg Chem. 2013;52(21):12223-12233.

54. Schulz K, Kroner A, David S. Iron efflux from astrocytes plays a role in remyelination. J Neurosci. 2012;32(14):4841-4847.

55. Duck KA, Connor JR. Iron uptake and transport across physiological barriers. Biometals. 2016;29(4):573-591.

56. Burkhart A, et al. Expression of iron-related proteins at the neurovascular unit supports reduction and reoxidation of iron for transport through the blood-brain barrier. Mol Neurobiol. 2016;53(10):7237-7253.

57. Moos T. Brain iron homeostasis. Dan Med Bull. 2002;49(4):279-301.

58. Jefferies WA, Brandon MR, Hunt SV, Williams AF, Gatter KC, Mason DY. Transferrin receptor on endothelium of brain capillaries. Nature. 1984;312(5990):162-163.

59. Wessling-Resnick M. Iron homeostasis and the inflammatory response. Annu Rev Nutr. 2010;30:105-122.

60. Nemeth E, et al. Hepcidin regulates cellular iron efflux by binding to ferroportin and inducing its internalization. Science. 2004;306(5704):2090-2093.

61. Andersen HH, Johnsen KB, Moos T. Iron deposits in the chronically inflamed central nervous system and contributes to neurodegeneration. Cell
Mol Life Sci. 2014;71(9):1607-1622.

62. Leibovitch EC, et al. Herpesvirus trigger accelerates neuroinflammation in a nonhuman primate model of multiple sclerosis. Proc Natl Acad Sci US A. 2018;115(44):11292-11297.

63. Absinta M, et al. Seven-tesla phase imaging of acute multiple sclerosis lesions: a new window into the inflammatory process. Ann Neurol. 2013;74(5):669-678.

64. Leibovitch E, et al. Novel marmoset (Callithrix jacchus) model of human Herpesvirus 6A and 6B infections: immunologic, virologic and radiologic characterization. PLoS Pathog. 2013;9(1):e1003138.

65. Sati $\mathrm{P}$, et al. In vivo quantification of $\mathrm{T}_{2}$ anisotropy in white matter fibers in marmoset monkeys. Neuroimage. 2012;59(2):979-985.

66. Reich DS, et al. Sample-size calculations for short-term proof-of-concept studies of tissue protection and repair in multiple sclerosis lesions via conventional clinical imaging. Mult Scler. 2015;21(13):1693-1704.

67. Absinta M, et al. Postmortem magnetic resonance imaging to guide the pathologic cut: individualized, 3-dimensionally printed cutting boxes for fixed brains. J Neuropathol Exp Neurol. 2014;73(8):780-788.

68. Guy JR, Sati P, Leibovitch E, Jacobson S, Silva AC, Reich DS. Custom fit 3D-printed brain holders for comparison of histology with MRI in marmosets. J Neurosci Methods. 2016;257:55-63.

69. Luciano NJ, Sati P, Nair G, Guy JR, Ha SK, Absinta $\mathrm{M}$, et al. Utilizing $3 \mathrm{D}$ printing technology to merge MRI with histology: a protocol for brain sectioning. JVis Exp. 2016;118.e54780. 